\title{
Modulation of Excess Electron Transfer through LUMO Gradients in DNA Containing Phenanthrenyl Base Surrogates
}

\author{
Pascal Roethlisberger, ${ }^{[a]}$ Veerabhadrarao Kaliginedi, ${ }^{[a]}$ and Christian, J. Leumann*[a]
}

\begin{abstract}
The modulation of excess electron transfer (EET) within DNA containing a dimethylaminopyrene (C-AP) as an electron donor and 5-bromouracil $\left({ }^{\mathrm{Br}} \mathrm{dU}\right)$ as a electron acceptor through phenanthrenyl pairs (phen-R) could be achieved by modifying the phenanthrenyl base surrogates with electron withdrawing and donating groups. Arranging the phenanthrenyl units to form a descending LUMO gradient increased the EET efficiency compared to the electron transfer through uniform LUMOs or an ascending LUMO gradient.
\end{abstract}

The well-defined double helical structure of DNA with the linear arranged base pairs represents a suitable scaffold for charge transfer and is therefore subject to intense studies in DNA damage,$^{[1]}$ sensors ${ }^{[2]}$ and applications in molecular electronics. ${ }^{[3]}$ The reductive electron transfer also called excess electron transfer (EET) in DNA is a process that is directed through the lowest unoccupied molecular orbital (LUMO) of the DNA bases. Investigations elucidated that the charge transfer over longer distances occurs via electron hopping mostly through thymine bases $\left(\mathrm{k}=10^{10} \mathrm{~s}^{-1}\right) \cdot{ }^{[4]}$ In earlier studies it was shown that the replacement of the natural base pairs by non-hydrogen bonding base surrogates with extended aromatic surfaces such as phenanthrene could have beneficial conducting properties and could overcome the physico-chemical limitations of the natural nucleobases. ${ }^{[5]}$ Regarding the reduction of such base surrogates the choice of the electron injector is crucial for the success of the experiments. Investigations by Grigorenko et. al. revealed that pyrene $\left({ }^{\mathrm{Py}} \mathrm{dU}, \mathrm{E}_{\mathrm{red}}{ }^{*}=-2.2 \mathrm{~V} \text { vs } \mathrm{NHE}\right)^{[6]}$ only enables a superexchange mechanism, whereas phenothiazine $\left(\mathrm{PTZ}, \mathrm{E}_{\mathrm{red}}{ }^{*}=\right.$ $-2.7 \mathrm{~V}$ vs $\mathrm{SCE})^{[7]}$ allowed to trigger the system into an electron hopping mechanism with a transport that spreads over longer distances. ${ }^{[5]}$ A photoexcitable dimethyl amino-pyrenyl donor attached to a deoxyuracil ( ${ }^{\mathrm{AP}} \mathrm{dU}, \mathrm{E}_{\mathrm{red}}{ }^{*}=-2.2 \mathrm{~V}$ vs $\mathrm{NHE}$ ) ${ }^{[8]}$ that exhibits suitable redox properties for long range charge transfer experiments was successfully used by Bätzner et. al. to inject an electron in hydroquinoline base surrogates. ${ }^{[9]}$

In this study, we investigated the EET through DNA containing phenanthrenyl base surrogates with different reduction potentials and LUMO energy levels. It is believed that the electron transfer within DNA can be modulated by the installation of a potential energy gradient. ${ }^{[10]}$ The predicted advantage of such a redox/LUMO gradient was envisioned to be the unidirectionality of the electron transfer and therefore a gain in efficiency. The installation of the different reduction potentials was deemed to be possible by the introduction of electron withdrawing $(\mathrm{CN})$ and donating $\left(\mathrm{NH}_{2}\right)$ groups at the 7-position of the phenanthrene (phen). The synthesis of the ${ }^{\mathrm{NH}_{2}}$ phen and phen phosphoramidites applicable for automated DNA synthesis was performed according to published procedures. ${ }^{[11]}$ The introduction of a cyano group required a palladium catalyzed substitution of the intermediate $\mathbf{9}$ with copper-(I)-cyanide. Tritiylation and phosphitilation of the ${ }^{\mathrm{CN}}$ phen $C$-nucleoside occurred under standard conditions and yielded the corresponding phosphoramidite 12 (see Scheme S3 in ESI). The redox properties of the building blocks were analyzed by cyclic voltammetry at the level of the free nucleosides 10 ( ${ }^{\mathrm{CN}}$ pen, $\mathrm{E}_{\text {red }}=$ $-1.63 \mathrm{~V}$ vs $\mathrm{Ag} / \mathrm{AgCl}), 13\left({ }^{\mathrm{NH}_{2}}\right.$ phen, $\mathrm{E}_{\text {red }}=-2.60 \mathrm{~V}$ vs $\left.\mathrm{Ag} / \mathrm{AgCl}\right)$ and 14 (phen, $E_{\text {red }}=-2.52 \mathrm{~V}$ vs $\mathrm{Ag} / \mathrm{AgCl}$ ). In addition, the density functional theory calculations (B3LYP/6-31G*) were found to correlate with the experimentally determined reduction potentials (see Figure $\mathrm{S} 1$ in $\mathrm{ESI}$ ).

a)
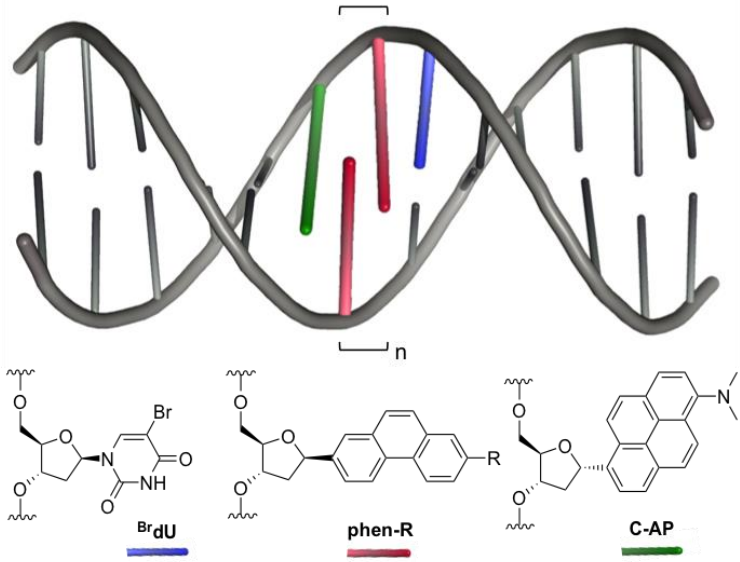

b)

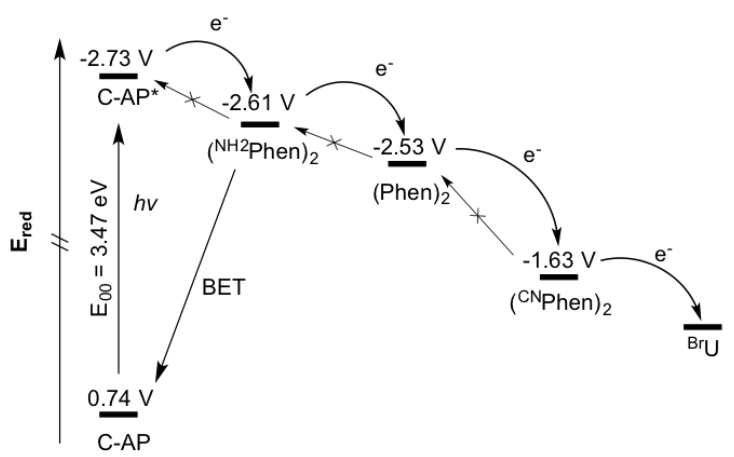

Figure 1. a) Schematic representation of the EET system, consisting of a photoexcitable $C$-AP donor opposite an abasic site, zipper like stacked phenanthrenyls and a 5-bromouracil $\left({ }^{\mathrm{Br} d U}\right)$ as electron acceptor. b) Representation of the energetics in the electron transfer process of the system consisting of a $C$-AP donor, the modified phenanthrenyls and the ${ }^{\mathrm{Br}} \mathrm{dU}$ acceptor.

In order to study the EET properties through phenanthrenyl base surrogates a $\alpha$ - $C$-nucleosidic dimethylamino-pyrene $(C$-AP) was synthesized that could intercalate well against an abasic site, which enables an efficient photo induced electron injection due to the close proximity to the phenanthrenyl stack. ${ }^{[12]}$ The synthesis involved a nucleosidation of the chloro Hofer sugar and a Gilman cuprate $^{[13]}$ of the 6 -bromo- $N, N$-dimethylpyren-1-amine, which was received from bromination, nitrification, reduction and dimethylation of the amine function of the pyrene (see Scheme $\mathrm{S} 1$ and Scheme S2 in ESI). According to cyclic voltammetry this 
donor (6) was found to have suitable reduction potential in the excited state $\left(\mathrm{E}_{\text {red }}{ }^{*}=-2.7 \mathrm{~V}\right.$ vs $\left.\mathrm{Ag} / \mathrm{AgCl}\right)$ to reduce all the phenanthrenyl units.

As an electron acceptor 5-bromouracil ( $\left.{ }^{\mathrm{Br}} \mathrm{dU}\right)$ was used, which releases a bromine anion $\left(\mathrm{Br}^{-}\right)$after encounter and capture a migrating electron that is injected into the DNA upon excitation of the $C$-AP at $420 \mathrm{~nm}$. The formed 5-uracyl radical abstracts a hydrogen form the 5' adjacent deoxyribose, which eventually affords alkali label products under aqueous conditions. ${ }^{[14]}$ The EET efficiency can then be evaluated by fragment analysis using polyacrylamide gel electrophoresis (PAGE) and control sequences as markers for the specific fragments (see figure 2). With the phosphoramidites of $C$-AP, phen, ${ }^{{ }^{C N}}$ phen, ${ }^{{ }^{N} H_{2}}$ phen and ${ }^{\mathrm{Br}} \mathrm{dU}$ a series of oligonucleotides were synthesized containing either a single (D1-D16) or triple (D17-D29) phenanthrenyl modifications between the pyrenyl donor and the electron acceptor. Thermal denaturing studies revealed that single phen modifications in general lead to a destabilization. On the other hand multiple phen modifications stabilized the duplex compared to the natural base pairs. This effect was observed in earlier studies with non-hydrogen bonding base surrogates and was found to be an enthalpy driven process induced by the increased hydrophobic interactions of such base surrogates. ${ }^{[11,15]}$ An expected decrease in stability was observed for duplexes containing electron donating groups and vice versa a stabilization of duplexes containing electron withdrawing groups.

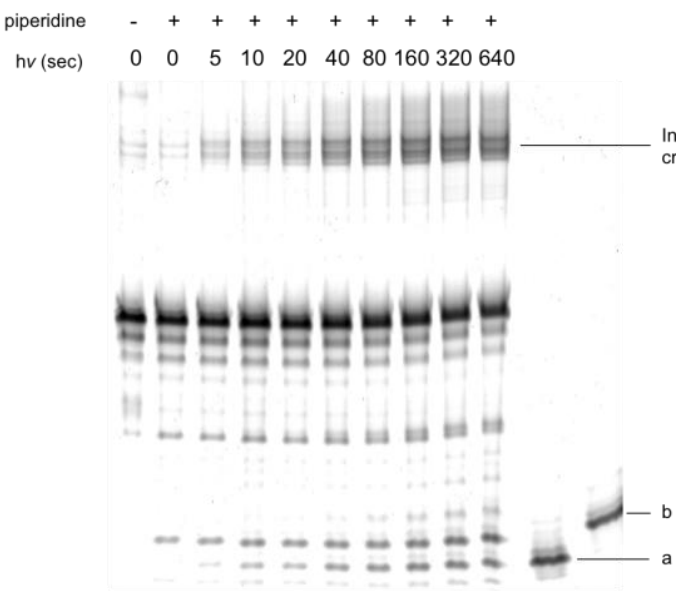

Figure 2. Representative denaturing PAGE showing the fragmentation of the duplex D8 after $640 \mathrm{sec}$ of irradiation at $420 \mathrm{~nm}$. Conditions: $4 \mu \mathrm{M}$ duplex, 10 $\mathrm{mM} \mathrm{NaH}_{2} \mathrm{PO}_{4}, 0.15 \mathrm{M} \mathrm{NaCl}, \mathrm{pH}$ 7.0. The duplex was exposed to the UV light for the indicated amount of time and analysed after subsequent piperidine treatment at $90^{\circ} \mathrm{C}$ for 30 min. Lane 1 contains the control under light exclusion and without piperidine treatment. The additional lanes show the specific fragments; a, $\mathrm{PO}_{4}$-ACGC-FAM; b, $\mathrm{PO}_{4}$-TACGC-FAM

Circular dichroism (CD) spectroscopy revealed that the secondary structure of natural DNA is not disturbed by the phenanthrenyl or pyrenyl modifications.

Initial EET experiments were performed with duplexes D1, D2, D3 lacking the donor or D4, D5, D6 without an acceptor. Both series did not show any fragment formation upon irradiation. In general a non-specific cleavage after piperidine treatment without irradiation occurred due to the applied heat. ${ }^{[16]}$ In addition to the specific short fragments a low mobility band occurred in sequences with ${ }^{\mathrm{NH}_{2}}$ phen and phen pairs. According to mass spectrometry the reaction product correlates to an intrastrand crosslink, as already observed in earlier EET studies with phen pairs (see Figure S5 and Figure S6 in ESI). ${ }^{[5]}$

Electron transfer through single phenanthrenyl (D8, D11, D14) pairs is less effective than EET through A/T (D7) base pairs due to the fact that thymine $(-0.95 \mathrm{eV}){ }^{[17]}$ exhibits a lower reduction potential compared to all the phenanthrenyl base surrogates. Additionally, in literature a suppression of hole transfer processes was found for duplexes with base mismatches ${ }^{[18]}$ and bulge positions ${ }^{[19]}$ suggesting that a slight perturbation of the base stack in the case of single phenanthrenyl pairs is accompanied with suppressing effects as well (see figure 3 ). ${ }^{[20]}$

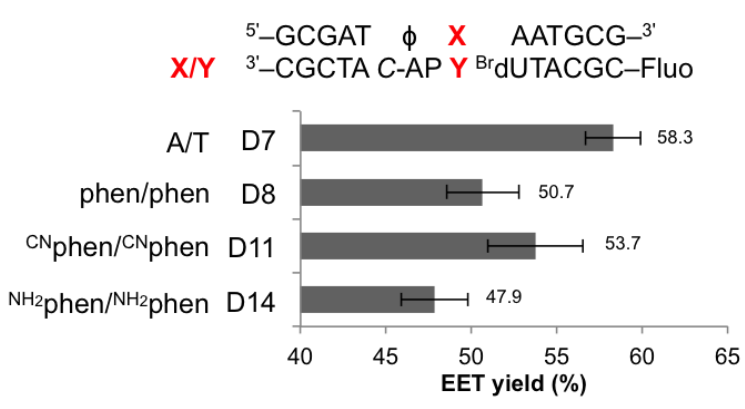

Figure 3. DNA cleavage yields for duplexes D7-D16 after 640 seconds irradiation at $420 \mathrm{~nm}$.

Comparing the EET yield of the different phenanthrenyl pairs, it was found that the efficiency of the electron transfer processes correlates with the reducibility of the phenanthrenyl base surrogates ( ${ }^{\mathrm{CN}}$ phen $>$ phen $>\mathrm{NH}_{2}$ phen). The differences in yield, however, were found to be within a $6 \%$ range. This could be explained by the fact that electron transfer over short distances can also occur via hole transfer and therefore lowers the influence of the reducibility in such processes. Analysis of the permutated phenanthrenyl sequences (D9, D10, D12, D13) show no statistically significant difference in EET yield and are therefore not further discussed (See Table S4 in ESI).

Extending the phenanthrenyl units from one to three consecutive incorporations for each strand allows for the installation of an electron gradient over longer distance. In this context, gel electrophoretic analysis of irradiated duplexes D17, D18, D19, D20, D21, lacking an electron donor, revealed different features: i) in general no major irradiation dependent strand cleavage occurred through three consecutive phen (D17) and ${ }^{\mathrm{CN}}$ phen (D19) base pairs; ii) installation of a LUMO gradient in an ascending manner produced an irradiation dependent fragmentation with a yield of up to $33.7 \%$ over 640 seconds without the use of an electron injector. It is assumed that the consecutive phenanthrenyl units can form an exciplex and absorb light at higher wavelength. An unexpected dominant strand cleavage product, with a lower mobility than the $5 \mathrm{mer}$, was observed for the ${ }^{\mathrm{NH}_{2}}$ phen containing duplex D18. It occurs in the dark as well as in a time dependent fashion upon irradiation. The same fragmentation pattern, but in much lower extent, was observed for duplex D21 having an ascending LUMO gradient with ${ }^{\mathrm{NH}}$ phen at the 5' end of the phen stack. It is not evident from these studies why this fragment is produced in the absence of irradiation at low temperatures $\left(4^{\circ} \mathrm{C}\right.$ storage). However, a possible explanation for the fragmentation is that a ground state reaction is enabled by an 
enlarged $\pi$-stack of ${ }^{\mathrm{NH} 2}$ phen units which could stabilize the resulting cationic species on the $5^{\prime}{ }^{\mathrm{NH}}$ phen in a similar way to what has been observed for guanine rich sequences in hole

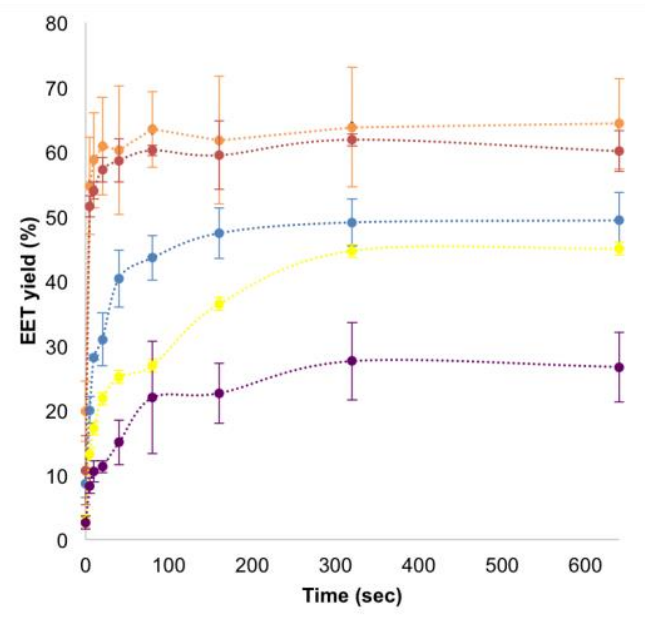

transfer $(\mathrm{HT})$ studies. ${ }^{[21]}$

Figure 4. EET from excited $C$-AP to ${ }^{\mathrm{Br}} \mathrm{dU}$ through multiple base surrogates as a function of time. (D27; $\bullet={ }^{\mathrm{CN}}$ phenphen ${ }^{\mathrm{NH}_{2}}$ phen), (D22; = TTT), (D23; $=$ phenphenphen), $\quad\left(\mathbf{D} 24 ; \bullet={ }^{\mathrm{CN}}\right.$ phen ${ }^{\mathrm{CN}}$ phen ${ }^{\mathrm{CN}}$ phen), $\quad(\mathbf{D 2 6} ; \bullet=$ $\mathrm{NH}_{2}$ phenphen ${ }^{\mathrm{CN}}$ phen). Conditions are given in the legend of Figure 2.

The EET efficiency through three consecutive A-T base pairs decreased by $-13.3 \%$ (compare D7 to D22), while the efficiency through three phen pairs remained the same (compare D8 and D23). On the other hand, the extension of the $\pi$-stack of ${ }^{C N}$ phen enhanced the EET yield by $+6.3 \%$ (compare D24 to D11). Comparing the cleavage product yield of homologous duplexes, it was observed that the electron transfer through ${ }^{\mathrm{CN}}$ phen (D24) is $10.7 \%$ more efficient than through unsubstituted phen (D23) and $15.1 \%$ higher than through A-T base pairs (D22). Interestingly the increased electron transfer yield through phen units coincides with an increased stability of the DNA duplexes (See ESI). It is believed that a favorable conformation of a stabilized duplex allows more efficient electron transfer as described in hole transfer studies by Wasielewski and co workers. ${ }^{[22]}$ The fact that the electron transfer efficiency is higher for phen stacks than for neutral A-T base pairs that have an intrinsic lower reduction potential is implying that the transfer process is not solely dependent on the LUMO energy of the participants.

Installation of a descending LUMO gradient as in D26 resulted in an EET yield that is higher by $4.2 \%$ compared to three ${ }^{\mathrm{CN}}$ phen (D24) or $15.0 \%$ higher compared to three consecutive phenanthrene residues (D23). A suppression of back electron transfer and charge recombination could be used to explain the increase in EET. ${ }^{[23]}$ Furthermore, an over two-fold lower transfer performance was observed for the ascending LUMO gradient (D27, 26.7\%) compared to the descending LUMO gradient (D26, $64.3 \%$ ), highlighting the importance of an exergonic process. Interestingly the EET yield through duplexes with mixed ascending and descending strands (D28 and D29) a relative high electron transfer yield ( $54.7 \%$ to $53.5 \%$ ) was observed, assuming that the electron transfer occurs not solely by electron hopping but also via electron tunneling to overcome the endergonic migrating steps.

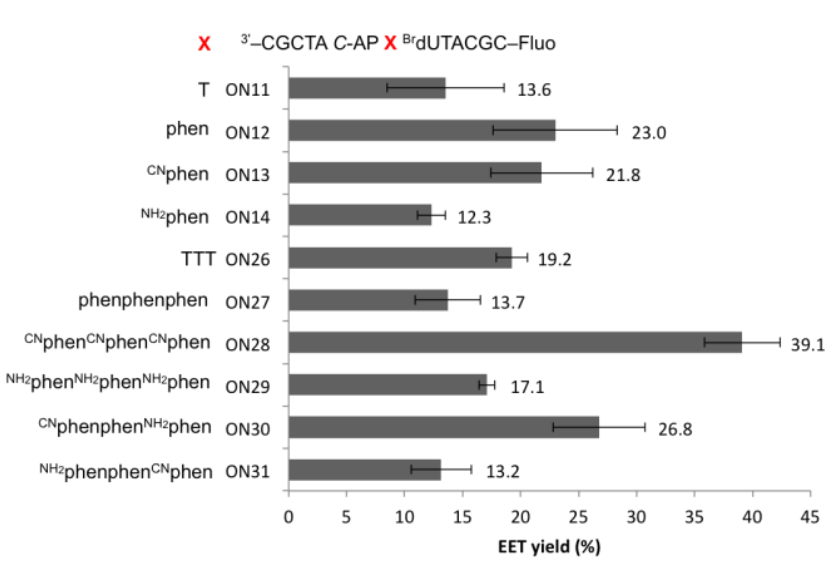

Figure 5. Comparison of DNA cleavage yields of single strands after 640 seconds irradiation at $420 \mathrm{~nm}$.

When the electron transfer through single strands was tested, a $\sim 50 \%$ loss of EET efficiency was observed in strands with single phen (ON12) and ${ }^{\mathrm{CN}}$ phen (ON13) incorporations while a loss of $\sim 75 \%$ of EET was determined for thymine (ON11) and ${ }^{\mathrm{NH} 2}$ phen (ON14) containing strands compared to their duplexes. Prolonging the distance between the donor and acceptor in single strands only led to a positive effect on the EET yield in the case of ${ }^{C N}$ phen (ON28-ON13, 19.3\%) but showed little effect in all other single strands. The observation that phenanthrene with an intrinsic higher reduction potential than thymine (-1.05 vs $\mathrm{Ag} / \mathrm{AgClO}_{4}$ or -1.86 vs $\left.\mathrm{NHE}\right)^{[17,24]}$ shows a higher electron transfer yield for long range migrations, indicate that the electron transfer efficiency cannot be explained by the driving force solely, even though the transfer yields increased with decreasing LUMO energies of the phen units. Okamoto et. al. introduced the concept of expanded aromatic systems in order to increase HTefficiencies by taking the advantage of the enhanced $\pi$-stacking properties. Experimental findings showed that the expanded aromatic hole mediator enhance the charge transfer over long distances (20 bp). ${ }^{[25]}$ Thus, it is believed that inter alia a high driving force, obtained by the installation of a descending LUMO gradient as well as the intrinsic large aromatic $\pi$-surfaces play a crucial role for the EET efficiency.

In summary the $C$-nucleosidic $C$-AP donor was found to be powerful and stable electron donor for EET experiments with an absorption band around $400 \mathrm{~nm}$ allowing for a selective excitation. Introducing modified phen base surrogates allowed the study of electron transfer through LUMO energy gradients. Although the interaction of the phenanthrenyl pairs within DNA could alter their LUMO levels to some extent, it is still possible to estimate the efficiency of the EET based on the LUMO levels of the isolated polyaromatic nucleosides. Indeed, an enhancement of electron transfer was found through a descending gradient compared to flat or ascending LUMO energy levels. The control of the electron transfer directionality widens the potential application of devices based on artificial DNA.

\section{Experimental Section}

All experimental details are provided in the Supporting information. 


\section{Acknowledgements}

Financial Support by the Swiss National Science Foundation (grantNo.:200020-130373)

Keywords: excess electron transfer $\cdot$ phenanthrene $•$ oligonucleotides $\cdot$ stacking interactions.

[1] aE. J. Merino, J. K. Barton, Biochemistry 2007, 46, 2805-2811; bM. E. Núñez, G. P. Holmquist, J. K. Barton, Biochemistry 2001, 40, 12465 12471; cD. J. Tan, R. K. Bai, L. J. C. Wong, Cancer Research 2002, 62 972-976.

[2] aA. L. Furst, M. G. Hill, J. K. Barton, Langmuir 2015, 31, 6554-6562; bH. Zhao, Z. Li, N. Y. Lee, J. S. Kim, E.-C. Lee, Current Applied Physics 2012 12, 1493-1496.

[3] aS. O. Kelley, J. K. Barton, N. M. Jackson, M. G. Hill, Bioconjugate Chem. 1997, 8, 31-37; bA. K. Boal, J. K. Barton, Bioconjugate Chem. 2005, 16 312-321; cE. M. Boon, J. K. Barton, Current Opinion in Structural Biology 2002, 12, 320-329; dS. O. Kelley, N. M. Jackson, M. G. Hill, J. K. Barton, Angew. Chem.-Int. Edit. 1999, 38, 941-945.

[4] M. J. Park, M. Fujitsuka, K. Kawai, T. Majima, J. Am. Chem. Soc. 2011 133, 15320-15323.

[5] P. Roethlisberger, F. Wojciechowski, C. J. Leumann, Chem.-Eur. J. 2013, 19, 11518-11521.

[6] N. A. Grigorenko, C. J. Leumann, Chem. Commun. 2008, 5417-5419.

[7] aT. Ito, A. Hayashi, A. Kondo, T. Uchida, K. Tanabe, H. Yamada, S. Nishimoto, Org. Lett. 2009, 11, 927-930; bT. Ito, A. Kondo, S. Terada, S. Nishimoto, J. Am. Chem. Soc. 2006, 128, 10934-10942.

[8] T. Ehrenschwender, W. Schmucker, C. Wellner, T. Augenstein, P. Carl, J. Harmer, F. Breher, H.-A. Wagenknecht, Chem.-Eur. J. 2013, 19 12547-12552.
[9] E. Batzner, Y. Liang, C. Schweigert, A. N. Unterreiner, H. A Wagenknecht, ChemPhysChem 2015, 16, 1607-1612.

[10] aT. Ito, R. Kurihara, N. Utsumi, Y. Hamaguchi, K. Tanabe, S.-i. Nishimoto, Chem. Commun. 2013, 49, 10281-10283; bM. Tanaka, K. Oguma, Y Saito, I. Saito, Chem. Commun. 2012, 48, 9394-9396.

[11] N. A. Grigorenko, C. J. Leumann, Chem.-Eur. J. 2009, 15, 639-645.

[12] T. Ito, S. E. Rokita, J. Am. Chem. Soc. 2004, 126, 15552-15559.

[13] S. Hainke, I. Singh, J. Hemmings, O. Seitz, J. Org. Chem. 2007, 72, 8811-8819.

[14] H. Sugiyama, Y. Tsutsumi, I. Saito, J. Am. Chem. Soc. 1990, 112, 6720 6721.

[15] aC. Brotschi, C. J. Leumann, Angew. Chem.-Int. Edit. 2003, 42, 16551658; bC. Brotschi, A. Haberli, C. J. Leumann, Angew. Chem.-Int. Edit. 2001, 40, 3012-3014.

[16] G. W. Grigg, Nucleic Acids Res. 1977, 4, 969-987.

[17] M. Hintze, K. O. Thiel, A. Vollmer, H. Brunner, C. Donner, Electrochimica Acta 2010, 55, 8135-8141.

[18] P. K. Bhattacharya, J. K. Barton, J. Am. Chem. Soc. 2001, 123, 86498656.

[19] D. B. Hall, J. K. Barton, J. Am. Chem. Soc. 1997, 119, 5045-5046.

[20] P. Roethlisberger, A. Istrate, M. J. Marcaida Lopez, R. Visini, A. Stocker, J. L. Reymond, C. J. Leumann, Chem. Commun. 2016.

[21] H. Sugiyama, I. Saito, J. Am. Chem. Soc. 1996, 118, 7063-7068.

[22] W. B. Davis, M. A. Ratner, M. R. Wasielewski, J. Am. Chem. Soc. 2001, 123, 7877-7886

[23] aT. Ito, Y. Hamaguchi, K. Tanabe, H. Yamada, S. Nishimoto, Angew. Chem.-Int. Edit. 2012, 51, 7558-7561; bF. Fakhari, Y. Y. K. Chen, S. E. Rokita, Chem. Commun. 2013, 49, 7073-7075.

[24] M. P. Scannell, D. J. Fenick, S. R. Yeh, D. E. Falvey, J. Am. Chem. Soc 1997, 119, 1971-1977.

[25] A. Okamoto, K. Tanaka, I. Saito, J. Am. Chem. Soc. 2003, 125, 5066 5071 